\title{
Tension pneumoperitoneum after surgery for endometrial cancer and hernia in a morbidly obese female: a case report
}

\author{
Bing-Sheng Lin ${ }^{1}$, Yan-Shen Shan², Wan-Chen Liư ${ }^{3}$, Chin-Han Wu${ }^{4}$, Pei-Ying Wu ${ }^{4}$ and Keng-Fu Hsu ${ }^{4 *}$
}

\begin{abstract}
Background: Obesity is a risk factor for the development of endometrial cancer and abdominal wall hernias. We report a case of tension pneumoperitoneum that developed after gynecological surgery and mesh repair of a ventral hernia.
\end{abstract}

Case presentation: A 57-year-old Asian Taiwanese woman with a body mass index of $52.9\left(\mathrm{~kg} / \mathrm{m}^{2}\right)$ underwent total abdominal hysterectomy and bilateral salpingo-oophorectomy due to endometrial cancer, and ventral herniorrhaphy with mesh due to ventral hernia. Tension pneumoperitoneum with severe dyspnea developed on postoperative day 14. Rather than performing emergency laparotomy as in visceral perforation, a transabdominal catheter was inserted to drain the intra-abdominal gas. This approach dramatically relieved the tension pneumoperitoneum and dyspnea. Our patient then recovered smoothly; the catheter was removed on postoperative 24 , and she was discharged on postoperative day 28. The clinical course of the endometrial cancer and repaired ventral hernia was well at the 1-year follow-up.

Conclusions: Tension pneumoperitoneum, which may result from the valve effect of unhealed abdominal mesh, could develop after gynecological surgery and hernia mesh repair in obese patients. Under these conditions, emergency drainage of the intra-abdominal gas by catheter insertion is sufficient to relieve the abdominal pressure and correct the conditions, while emergency laparotomy as in visceral perforation is unnecessary and may increase patient morbidities.

Keywords: Tension pneumoperitoneum, Endometrial cancer, Hernia, Obesity

\section{Background}

A high body mass index (BMI) is an established risk factor for the development of endometrial cancer [1]. Obesity predisposes patients to abdominal wall hernias. Obese patients with a higher BMI undergoing abdominal wall repair often have increased complications, including abdominal compartment syndrome, seroma, hematoma, infectious morbidity, and dehiscence, which typically occur early in the postoperative period [2, 3].

The accumulation of free intra-abdominal air with elevated intra-abdominal pressure is known as tension pneumoperitoneum (TP). TP may result in an elevation of the diaphragm, which reduces lung volume and compresses

\footnotetext{
* Correspondence: d5580@mail.ncku.edu.tw

${ }^{4}$ Department of Obstetrics and Gynecology, National Cheng Kung University Hospital, College of Medicine, National Cheng Kung University, 138 Sheng Li Road, Tainan 704, Taiwan

Full list of author information is available at the end of the article
}

the inferior vena cava, which in turn reduces the venous return and cardiac output and can result in aortic occlusion [4]. Various etiologies have been reported, including gastrointestinal perforation, barotrauma in ventilated patients, and diaphragmatic hernia repair [5]. Here, we report the case of a morbidly obese woman with TP that developed after surgery for endometrial cancer and the mesh repair of a ventral hernia. Rather than performing emergency laparotomy as in visceral perforation, she received drainage of the intra-abdominal gas by a transabdominal catheter which relieved the TP and her dyspnea.

\section{Case presentation}

A 57-year-old gravida 6, para 3, Asian Taiwanese woman presented to our hospital for scheduled surgery due to endometrial cancer. At admission, her body temperature was $36.8{ }^{\circ} \mathrm{C}$, pulse rate 78 beats/minute, respiratory rate 
20/minute, and blood pressure 136/98 mmHg. Her family and environmental history were unremarkable. She did not receive surgery before nor take any medication for systemic disease, such as diabetes mellitus or hypertension. She was a housekeeper. She did not smoke tobacco or consume alcohol. On physical examination, she had normal breathing sound, without wheezing or crackle, and a regular heartbeat without murmur. Her abdomen was soft with normoactive bowel sound on auscultation. Her extremities were freely moveable without edema. She exhibited severe morbid obesity (body weight, $128.4 \mathrm{~kg}$; height, $155.7 \mathrm{~cm}$; BMI, $52.9 \mathrm{~kg} / \mathrm{m}^{2}$ ). Her daily activities were independent most of the time, except for some episodes of exertional dyspnea. Computed tomography (CT) revealed a ventral hernia with a large fascial defect (Fig. 1). There were no clinical symptoms, such as abdominal pain or tenderness. She underwent total abdominal hysterectomy, bilateral salpingo-oophorectomy, and ventral herniorrhaphy with Goretex mesh (GORE DUALMESH ${ }^{\circ}$ ).

Her early postoperative course was smooth. She consumed water on postoperative day (POD) 3, a clear-liquid diet on POD5, and a semi-liquid diet on POD7. She began bedside ambulation, and half of her stitches were removed on POD10. However, progressive dyspnea was noted after bedside ambulation. Intra-abdominal free air with elevation of right-side hemidiaphragm was noted by chest X-ray (Fig. 2a) on POD14. There was no abdominal tenderness or rebounding pain, but there was moderate abdominal distension. She was kept under close observation and began nil by mouth (NPO) for possible visceral perforation.

Two days later (POD16), severe dyspnea and extensive abdominal distention were noted. Her respiratory rate was elevated to 26/minute with accessory muscle use. Arterial blood gas analysis (under simple $\mathrm{O}_{2}$ mask, $10 \mathrm{~L} /$ minute)

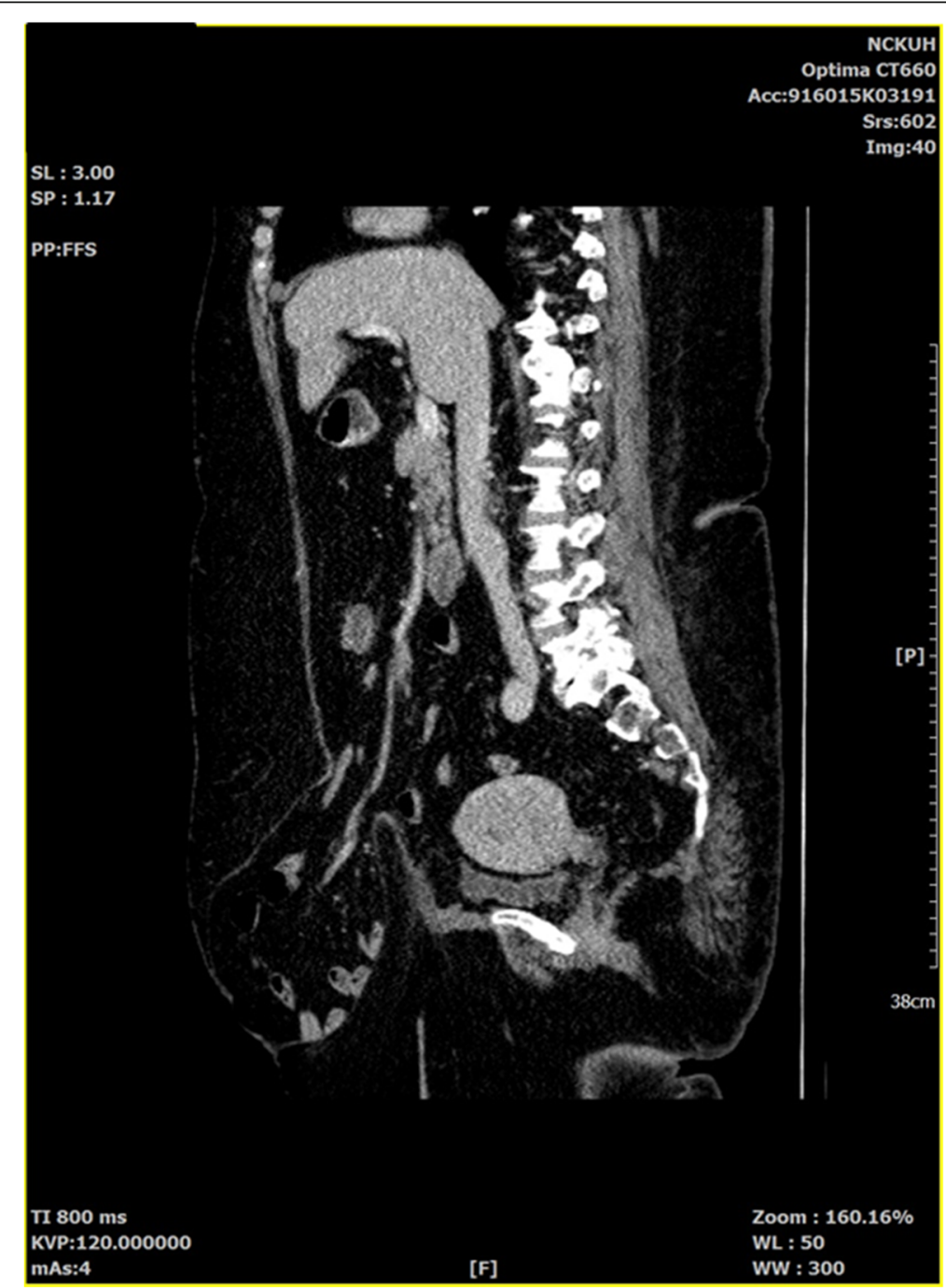

Fig. 1 Computed tomographic image showing a large ventral hernia sac 

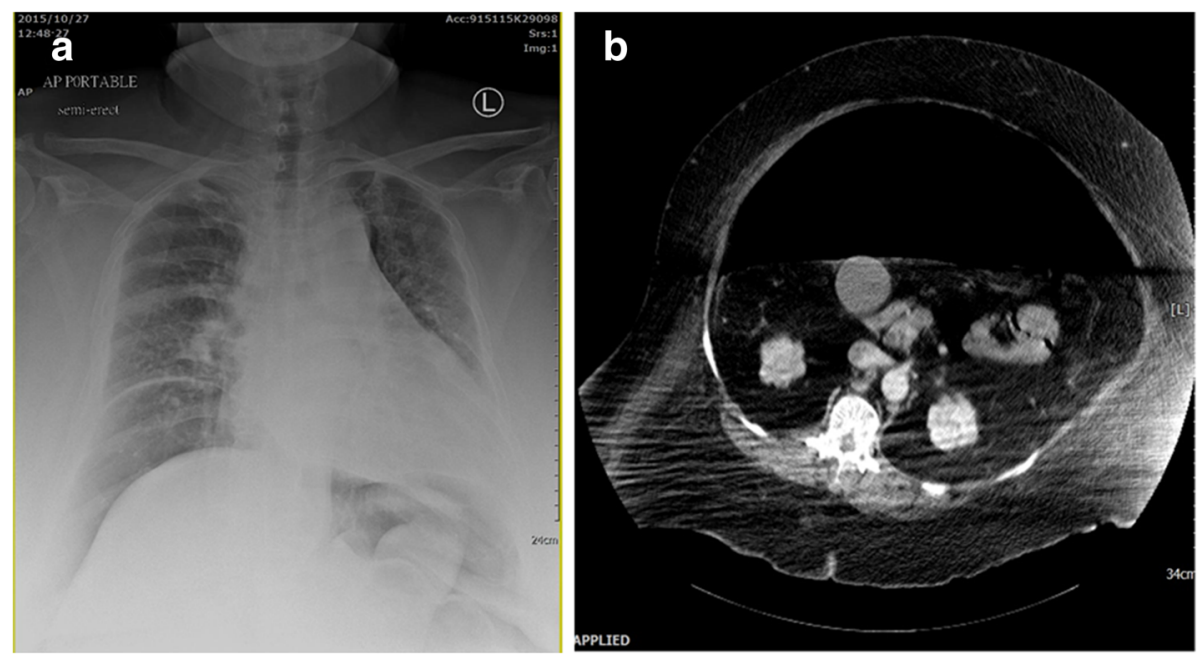

Fig. 2 a Chest X-ray demonstrating an elevated diaphragm and suspicious pneumoperitoneum. b Computed tomography scan, transverse view. Massive free air and minimal ascites were noted in the abdomen

showed: $\mathrm{pH}, 7.42$; partial pressure of carbon dioxide in arterial blood $\left(\mathrm{PaCO}_{2}\right), 44.8(\mathrm{mmHg})$; partial pressure of oxygen in arterial blood $\left(\mathrm{PaO}_{2}\right), 86.2(\mathrm{mmHg})$; bicarbonate $\left(\mathrm{HCO}_{3}-\right), 28.5(\mathrm{mmol} / \mathrm{L})$; blood oxygen saturation $\left(\mathrm{SpO}_{2}\right)$, 96.7\%; and blood pressure, 102/75 $\mathrm{mmHg}$. Laboratory tests revealed leukocytosis with a white blood cell (WBC) count of 12,900/uL, hemoglobin $(\mathrm{Hb}) 12.6 \mathrm{~g} / \mathrm{dL}$, and platelet count 310,000/uL. Her C-reactive protein (CRP) was elevated at $165.7 \mathrm{mg} / \mathrm{dL}$. Serum creatinine was 0.99 $\mathrm{mg} / \mathrm{dL}$, while serum sodium was $131 \mathrm{mmol} / \mathrm{L}$, and potassium was $3.9 \mathrm{mmol} / \mathrm{l}$. A physical examination showed severe abdominal distension without rebound pain. A pelvic examination demonstrated good healing of the vaginal cuff without leakage. CT showed a large amount of intra-peritoneal free air with little ascites (Fig. 2b). Because clinical visceral perforation was less likely, under the impression of pneumoperitoneum, in this emergent situation, an 8-Fr. pigtail catheter was inserted under CT guidance for decompression. A massive amount of gas was drained. Subsequently, her dyspnea and abdominal distention dramatically subsided. She re-started oral intake on POD20, and the abdominal pigtail catheter was removed on POD24 after no more free air was revealed by chest X-ray. No further abdominal distention was noted, and she was discharged on POD28. The clinical course of the endometrial cancer and repaired hernia were well at the 1-year follow-up.

\section{Discussion}

In our case, the TP may have resulted from the valve effect of unhealed hernia mesh. Unlike the TP caused by gastrointestinal perforation which needs emergency laparotomy to repair the perforation, it was unnecessary for our patient. The drainage of intra-abdominal free air is sufficient to improve this condition. The most common iatrogenic cause of pneumoperitoneum is abdominal surgery. Postoperative pneumoperitoneum is usually absorbed within 2 weeks. When pneumoperitoneum progresses with increasing intra-abdominal pressure, hemodynamic and ventilatory compromise might occur, and the condition results in TP. Dyspnea and venous congestion in the lower extremities might be noted during the physical examination. Similar to tension pneumothorax, emergency percutaneous needle decompression is needed [6]. When managing hollow organ perforation-related TP, a venous catheter may be percutaneously inserted to stabilize the patient's vital signs and to bridge the time to the start of the emergency operation [5].

Ventral hernia mesh repair may reduce tension on the abdominal wall, resulting in less recurrence compared with a simple suture repair. Various complications associated with hernia repair have been reported [2, 3]. However, there are few reports in the literature regarding $\mathrm{TP}$ as a complication related to hernia repair. In our case, abdominal free air was noted on POD14 after ambulation. The most plausible cause of TP was that air may have entered our patient's abdominal cavity via the unhealed abdominal wound, while the one-way valve effect developed due to morbid obesity. When reviewing the series of CT scans done on POD16, we found that fluid had accumulated from the mesh extending to the superficial wound (Fig. 3a-d), indicating the formation of a tract that allowed air to enter her abdominal cavity. When our patient began to ambulate, the thick abdominal subcutaneous fat and muscle detached from the mesh. This effect may have caused the formation of a small tract extending 

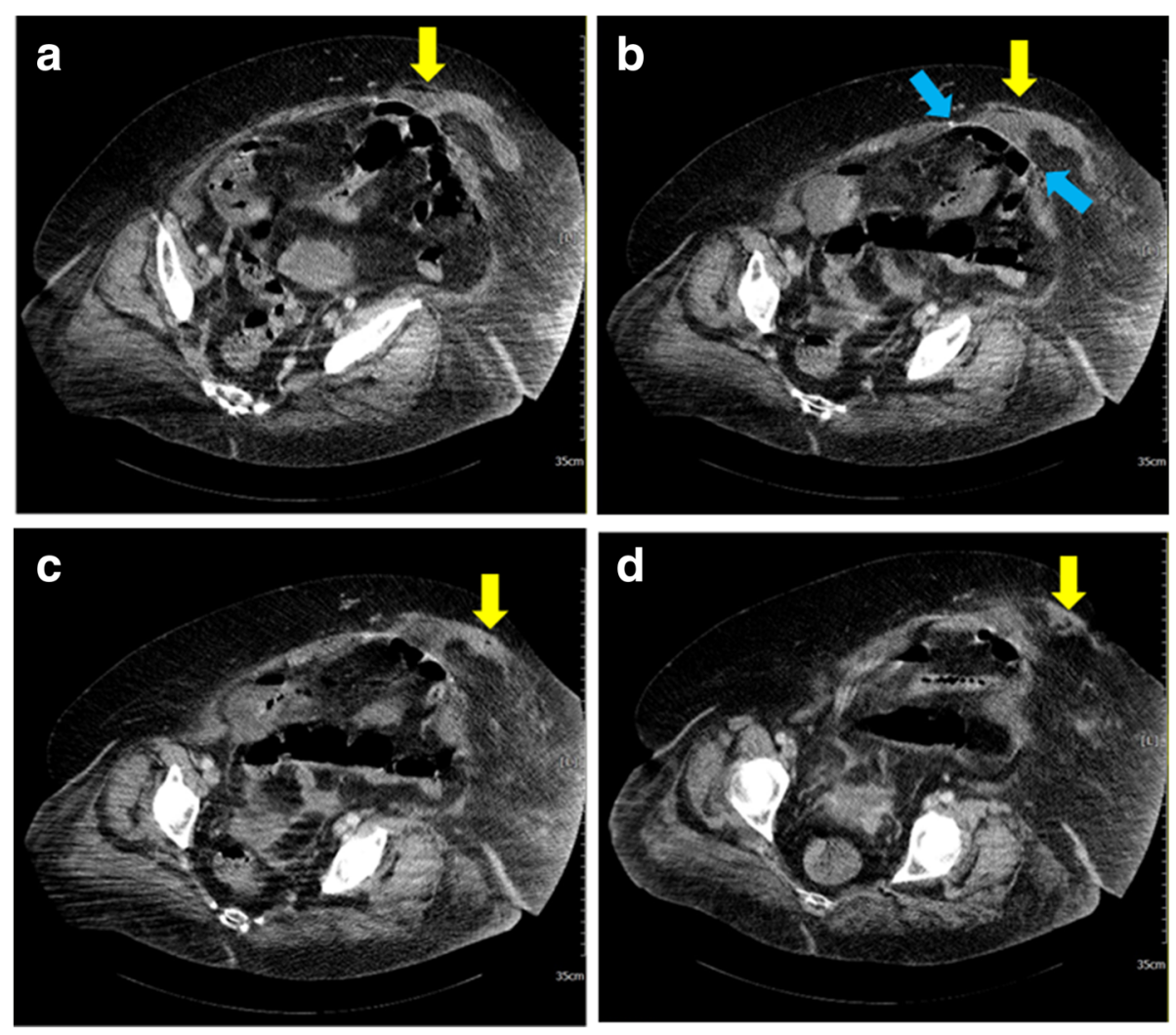

Fig. 3 Computed tomography scan, contrast-enhanced transverse view, cranial (a) to caudal (d). Series of subcutaneous fluid accumulation (yellow arrows) extending from the mesh (between blue arrows) to the superficial incision wound

from the superficial wound to the subcutaneous fat and mesh and into her abdominal cavity. When she exhaled, an upward movement of her diaphragm created negative pressure and caused a small amount of gas to enter her abdominal cavity. When she inhaled, although the downward movement of her diaphragm created a relative positive intra-abdominal pressure, her bowel may have sealed the tract, resulting in the prevention of free air escaping from her abdominal cavity. When she was lying down, the subcutaneous fat and muscle re-attached to the mesh, preventing the free air from leaving her abdominal cavity. Due to the valve effect, air accumulated gradually over several days and finally developed into TP.

Unlike the TP caused by gastrointestinal perforation, it was unnecessary for our patient to receive emergency laparotomy to repair the perforation. The drainage of intra-abdominal free air is sufficient to improve this condition. As the abdominal wound healed, the gas route sealed gradually.

\section{Conclusions}

Most surgery-related cases of pneumoperitoneum resolve spontaneously within 2 weeks after surgery. Caution should be taken if the pneumoperitoneum occurs or progresses 2 weeks after surgery in an obese patient with a mesh-repaired hernia. If accompanied by dyspnea, abdominal distention, and hemodynamic change, the possibility of TP should be considered, and emergency drainage of the intra-abdominal gas is needed. Emergency laparotomy is unnecessary for this condition.

\section{Abbreviations}

BMl: Body mass index; CT: Computed tomography; POD: Postoperative day; TP: Tension pneumoperitoneum

\section{Acknowledgements}

None.

\section{Funding}

None.

\section{Availability of data and materials}

Relevant data and supporting materials will be made available on request.

\section{Authors' contributions}

BSL, YSS, PYW, and KFH participated in patient care. WCL analyzed the X-ray data. BSL and YSS wrote the manuscript. CHW and KFH revised and edited the manuscript. All authors have read and approved the final manuscript.

Ethics approval and consent to participate

We have obtained a signed consent form from our patient that will be filed as part of our records. 


\section{Consent for publication}

Written informed consent was obtained from the patient for publication of this case report and any accompanying images. A copy of the written consent is available for review by the Editor-in-Chief of this journal.

\section{Competing interests}

The authors declare that they have no competing interests.

\section{Publisher's Note}

Springer Nature remains neutral with regard to jurisdictional claims in published maps and institutional affiliations.

\section{Author details}

'Department of Family Medicine, National Cheng Kung University Hospital, College of Medicine, National Cheng Kung University, Tainan, Taiwan. 2Department of Surgery, National Cheng Kung University Hospital, College of Medicine, National Cheng Kung University, Tainan, Taiwan. ${ }^{3}$ Department of Radiology, National Cheng Kung University Hospital, College of Medicine, National Cheng Kung University, Tainan, Taiwan. ${ }^{4}$ Department of Obstetrics and Gynecology, National Cheng Kung University Hospital, College of Medicine, National Cheng Kung University, 138 Sheng Li Road, Tainan 704, Taiwan.

Received: 30 June 2017 Accepted: 24 January 2018

Published online: 20 March 2018

\section{References}

1. Reeves KW, Carter GC, Rodabough RJ, Lane D, McNeeley SG, Stefanick ML, Paskett ED. Obesity in relation to endometrial cancer risk and disease characteristics in the Women's Health Initiative. Gynecol Oncol. 2011;121(2):376-82.

2. Fischer JP, Wink JD, Nelson JA, Kovach SJ 3rd. Among 1,706 cases of abdominal wall reconstruction, what factors influence the occurrence of major operative complications? Surgery. 2014;155(2):311-9.

3. Regner JL, Mrdutt MM, Munoz-Maldonado Y. Tailoring surgical approach for elective ventral hernia repair based on obesity and National Surgical Quality Improvement Program outcomes. Am J Surg. 2015;210(6):1024-9.

4. Chan SY, Kirsch CM, Jensen WA, Sherck J. Tension pneumoperitoneum. West J Med. 1996;165(1-2):61-4.

5. Symeonidis N, Ballas K, Pavlidis E, Psarras K, Pavlidis T, Sakantamis A. Tension pneumoperitoneum: a rare complication of upper gastrointestinal endoscopy. JSLS. 2012;16(3):495-7.

6. Chiapponi C, Stocker U, Körner M, Ladurner R. Emergency percutaneous needle decompression for tension pneumoperitoneum. BMC Gastroenterol. 2011;11:48.

\section{Submit your next manuscript to BioMed Central and we will help you at every step:}

- We accept pre-submission inquiries

- Our selector tool helps you to find the most relevant journal

- We provide round the clock customer support

- Convenient online submission

- Thorough peer review

- Inclusion in PubMed and all major indexing services

- Maximum visibility for your research

Submit your manuscript at www.biomedcentral.com/submit
Biomed Central 